\title{
PHOTOGRAMMETRIC EVALUATION OF MULTI-TEMPORAL FIXED WING UAV IMAGERY
}

\author{
E. Gülch \\ University of Applied Sciences Stuttgart, Department of Geomatics, \\ Computer Science and Mathematics, Schellingstr. 24, 70174 Stuttgart, Germany \\ eberhard.guelch@hft-stuttgart.de
}

Commission I, WG I/V

KEY WORDS: UAVs, Photogrammetry, Image Quality, Aerial Triangulation, DEM/DTM, Orthoimage

\begin{abstract}
:
Several flights have been undertaken with PAMS (Photogrammetric Aerial Mapping System) by Germatics, Germany, which is briefly introduced. This system is based on the SmartPlane fixed-wing UAV and a CANON IXUS camera system. The plane is equipped with GPS and has an infrared sensor system to estimate attitude values. A software has been developed to link the PAMS output to a standard photogrammetric processing chain built on Trimble INPHO. The linking of the image files and image IDs and the handling of different cases with partly corrupted output have to be solved to generate an INPHO project file. Based on this project file the software packages MATCH-AT, MATCH-T DSM, OrthoMaster and OrthoVista for digital aerial triangulation, DTM/DSM generation and finally digital orthomosaik generation are applied.

The focus has been on investigations on how to adapt the "usual" parameters for the digital aerial triangulation and other software to the UAV flight conditions, which are showing high overlaps, large kappa angles and a certain image blur in case of turbulences. It was found, that the selected parameter setup shows a quite stable behaviour and can be applied to other flights. Investigations have been performed to improve the image quality estimates by the PAMS software and extend it to whole images. This gives the user a reliable basis when deciding on rejecting images with low quality for the follow-up process. Flights over the same area at different times have been compared to each other. The major objective was first to see, on how far differences occur relative to each other, without having access to ground control data, which would have a potential for applications with low requirements on the absolute accuracy. In a second stage the results are compared to GPS measurements on the ground.

The results show, that there are influences of weather and illumination visible. The "unusual” flight pattern, which shows big time differences for neighbouring strips has an influence on the AT and DTM/DSM generation. The results obtained so far do indicate problems in the stability of the camera calibration and a need for some GCPs. It is clearly recommended to use at least a moderate number of GCPs for all projects, independent on the application, as the GPS data of the sensor does not proof to be sufficient.
\end{abstract}

\section{INTRODUCTION}

The HFT Stuttgart has purchased in 2009 the UAV PAMS (Photogrammetric Aerial Mapping System) offered by Germatics GmbH, Germany (Germatics, 2009, 2011). This system is based on the SmartPlane fixed-wing UAV and a CANON IXUS camera system. The plane is equipped with GPS and has an infrared sensor system to estimate attitude values.

The objectives are usage for thesis work, research and eventually for student exercises.

Whereas at the beginning the research focus has been on developing and investigating camera calibration issues (Brinker, 2008) and the usage of a predecessor of the UAV, the major attention has been put on connecting to standard photogrammetric processing software (here Trimble INPHO software (Trimble, 2011) which is available at HFT and investigations on how to adapt the software parameters to the typical flight patterns and conditions of this UAV (Ziegler, 2011). The PAMS system has built-in software packages for "Quick mosaic" and "Air mosaic" generation, which are certainly useful already for many visual applications and are the input to the processing service offered by the company, but which do not allow to directly link to standard photogrammetric processing software.

The output of the PAMS software version of HFT contains the parameters of a coarse aerial triangulation and the camera calibration parameters that can be imported into Trimble INPHO software (Trimble, 2011) project files. The linking of the image files and image IDs and the handling of different unusual cases with partly corrupted output will have to be solved to generate a Trimble INPHO project file. Based on the generated project file the software packages MATCH-AT, MATCH-T DSM, OrthoMaster and OrthoVista for digital aerial triangulation, DTM/DSM generation and finally digital orthomosaik generation are used for various investigations.

One focus has been on how to adapt the "usual” parameters for the digital aerial triangulation and other software to the UAV flight conditions, which are showing high overlaps, large kappa angles and a certain image blur in case of turbulences. It was of additional interest on how those parameters can be useful for other flights as well.

Investigations have been performed to improve the currently available image quality estimates by the PAMS software (AirMosaic module) and extend it to whole images. This is expected to give the user a more reliable basis when deciding on rejecting images with low quality for the follow-up process.

Flights over the same area at different times have been compared to each other. One major objective was here to see, on how far differences occur relative to each other, without having access to ground control data, which would have a 
potential for applications with low requirements on absolute accuracy.

Based on manual GPS measurements of a higher number of GCPs some overall quality estimate of the flights can be generated which are presented at the end.

\section{BACKGROUND}

The first UAVs have been developed right after World War I. Besides the traditional dominant military applications it is observed, that during the last decade more and more commercial, non-military applications are offered world-wide. This comprises vendors and service providers. This trend is acknowledged by ISPRS with a first Inter-commission WG I/V on "Autonomous vehicle navigation" (ISPRS, 2004) and now with the Inter-commission WG I/V "UVS for mapping and monitoring applications” (ISPRS, 2011), organizing this conference.

The UAV platforms can be classified into Airships UAV Fixed Wings UAV, Powered Paraglider UAV and Rotary Wings UAV (Everaerts, 2006). Earlier a major focus of the systems with low weight (also called Micro-UAVs) has been on pure image acquisition. A photogrammetric evaluation was not envisaged. This has been changed in recent years. The strong miniaturization of GPS, IMU sensors and cameras in connection with rather high quality performance has opened the path for higher quality photogrammetric processing tasks.

UAV vendors do not only offer RGB digital cameras, but also offer or plan to offer NIR sensors, thermal sensors or day-light and twilight sensors for special surveillance and monitoring tasks. A video-downlink for quick visual analysis is partly available. Some UAV systems do offer long operating time and high pay load but are usually only manageable with specially trained and certified personnel and are limited in the application area due to legal reasons.

Various helicopter, quadcopter or oktocopter systems are offered with different pay-load features, up to laser-profilers or laser-scanners. The usage can be heavily restricted due to wind conditions, especially with low-cost and low-weight devices. Many of those systems are definitely applicable in huge indoor rooms as well as in favorable outdoor conditions. The HFT Stuttgart decided to go for fixed-wing UAV with small payload and robust handling for the envisaged application areas and due to budget limitations.

The fixed-wing Micro-UAVs do not really compete with traditional photogrammetric flights, but offer interesting options for small areas, where the costs of traditional flights are simply too high. There is no need for a real runway, flight altitudes are usually only some $200 \mathrm{~m}-300 \mathrm{~m}$ above ground and thus below cloud coverage. With special control sensors and intelligent software also non-expert users might be able to perform a photogrammetric flight. These systems do offer autonomous flights following a defined flight planning. By linking the sensor images and meta-data to the usual digital photogrammetric processing chain, new applications with photogrammetric products like Digital Terrain Models (DTMs) and Digital Surface Models (DSMs) as input for orthophoto generation are possible. Some companies do also not only act as vendors of UAV systems but do also offer services for different application areas (cf. e.g. Table 1).

The company Germatics GmbH (Germatics, 2009, 2011) does not only offer a Micro-UAV (Personal Aerial Mapping System, PAMS) since beginning of 2009, but also services for generation of orthophotos and DTMs/DSMs and other products. The company Mavinci (Mavinci, 2011) offers several types of fixed-wing UAVs with a pay load of partly more than $1 \mathrm{~kg}$. Due to higher weight there is a need for more operators and there are certain limitations in usage due to flight regulations. Mavinci offers links to photogrammetric software for orthomosaic and DSM generation. Gatewing (Gatewing, 2011) offers services very similar to Germatics as announced in late 2009. The fixedwing system (Gatewing, X100) can be combined with services for producing DSMs and orthomosaiks.

The security aspects do play an important role and the usage by untrained personal without special licenses is a pre-requisite for usage with students. The selected system by HFT was the Personal Aerial Mapping System (PAMS) by the Germactics $\mathrm{GmbH}$, Germany which is described in the next section.

Table 1: Examples for application areas for UAVs

\begin{tabular}{|l|}
\hline Monitoring, inspection, documentation \\
\hline Disaster monitoring and prevention \\
\hline Planing tasks in region, city and rural areas \\
\hline Landscape ecology \\
\hline Farming \\
\hline Private sector \\
\hline Archaeology \\
\hline Hydrology \\
\hline Forestry \\
\hline Surveillance \\
\hline
\end{tabular}

\section{PERSONAL AERIAL MAPPING SYSTEM}

\subsection{Description of the PAMS system}

The PAMS System (Germatics, 2009, 2011) consists of a fixedwing UAV (SmartOne, cf. Figure 1) and a controlling unit on ground. The wingspread is $1.2 \mathrm{~m}$. The electric motor and the battery pack used allow missions of about 45 minutes duration with an average speed of $15 \mathrm{~km} / \mathrm{h}$. An autopilot system integrated into the UAV allows autonomous flights of a predefined flight path. The weight with camera is about $1.1 \mathrm{Kg}$. The camera used is a calibrated IXUS 70 (7Mpix, 3072x2304 $\mathrm{pel}^{2}$ ) for RGB imagery stored on flash card 2GB. The pixel size is about $1.9 \mu \mathrm{m}$. The camera focus is fixed to infinity during the flight.

The plane was designed to be handled by one person only. There is no special need for a runway, but some open ground for start and landing phases. The controlling unit consists of a radio device, a classical remote control for semi-automatic control or manual interaction in case of emergencies and a laptop for the flight planning and monitoring. Inter-visibility is a pre-requisite for this UAV, which allows an operating range from the ground unit is about $500 \mathrm{~m}$ to $2500 \mathrm{~m}$. The average height above ground can be chosen between 150 and $600 \mathrm{~m}$, but is in the described flights mostly set to 150 to $200 \mathrm{~m}$, which allows about 4.6-6.2cm GSD on the ground.

There are several steps of the preparation and performance of a typical flight mission. They are built on the ground station HW/SW and the remote flight control device. After some short training, those tasks can in most parts reliably solved even by untrained personnel. In the following the experiences from some of the flights performed so far are summarized.

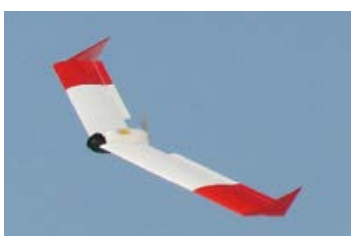

Figure 1: PAMS UAV (Germatics GmbH, 2009). 


\subsection{Experiences from flight missions and preparation tasks}

Step 1 is to build-up the device and the ground station. With two persons this can be reliably done in 10 to 15 minutes. The plane parts have to be connected, the camera fixed, as well as all the cables have to be connected and the H/W has to be checked. The ground station has to be built up and the radio link has to be checked. An overview on a typical flight is given in Table 2). In the next step the flight planning has to be done. The flight planning software is very user friendly, but for the planning an orthophoto of the area in question has to be organized externally for better visual setting. It has been shown advantageous, that the flight plan can be still finalized on site to adapt to wind directions and other external conditions. The basic input parameters are the area definition (width, height), strip directions and flying height above ground. A base point for the plane has to be defined, which is used as waiting point before and after the photogrammetric flight mission. After the planning is finalized and all systems are active, the UAV can be started.

The user has the chance to interact in full manual mode, or to use the guided mode (Auto mode 1), with aircraft sensors trying to level the plane once airborne, which allows even an untrained user to start and later to land the UAV safely. For the start itself, it is, at least for rather untrained users, favorable to have another person starting the plane by hand, while the operator takes care of the remote control device. For trained personnel it is possible to perform both tasks. After the start, the integrated sensors allow an automated flight to the start position and the planned height. If all systems show status "OK", the operator starts the photogrammetric flight mission which is flown autonomously (Auto mode 2) by the UAV. During the mission it has been found advantageous to regularly check in the air and/or on the ground station the flight path and watch out for objects (birds, kites, balloons etc.) which might require immediate emergency reactions from the operator. During the autonomous flight the impact by wind is monitored and the UAV tries to counteract, if needed, to fulfill the planned parallel flight lines and exposure stations. All meta-data is sent to the ground station and recorded. The user has full overview on camera-, GPS- and battery status as well as on the flown parts of the planned block. After the photogrammetric flight mission, the UAV moves back to the waiting position defined in the planning phase and the operator performs the landing semiautomatically (Auto mode 1) or manually. It has been found, that some training time is needed not only for the starting phase, but especially also for the landing phase. For both it is advantageous to use Auto mode 1. The landing area chosen so far was grassland with grass of various heights.

Table 2: Flight parameters (BezF6, June 8th 2010).

\begin{tabular}{|l|l|}
\hline Preparation time & About $10 \mathrm{~min}(2$ persons) \\
\hline Flight planning & Area 119x250 $\mathrm{m}^{2}$ \\
& Nominal GSD 4.6cm \\
\hline Start & Auto mode 1 \\
\hline Photogrammetric flight & Flight time $5 \mathrm{~min} 39 \mathrm{sec}$ \\
mission & 88 images \\
& Average speed $14.61 \mathrm{~m} / \mathrm{s}$ \\
& Average wind speed $(4.11 \mathrm{~m} / \mathrm{s})$ \\
\hline Landing & Auto mode 1 \\
\hline
\end{tabular}

\subsection{PAMS UAV Evaluation Software}

After the image and meta-data files from the SD card of the UAV camera has been imported into the ground station, the PAMS evaluation software can be applied. The PAMS UAV evaluation software has actually two parts, the $1^{\text {st }}$ level is integral part of the UAV system, the $2^{\text {nd }}$ level is offered as service and not included in the UAV system (cf. Table 3).

The $1^{\text {st }}$ Level Ground processing software is divided in two modules. The "QuickMosaic" software allows a quality control right after the flight on site and a so called quick mosaic of the images is produced. The "AirMosaic" software allows the production of a so called airmosaic (or "dirty" orthomosaic), based on an automated AT, DTM/DSM generation and orthomosaic generation down to resolution level 3. The software allows to check the quality of each image and to exclude highly blurred images from further processing (cf. Figure 2) The HFT PAMS SW version allows the export of the raw images (jpeg format) and the GPS/attitude data derived from the AirMosaic software combined with the camera calibration parameters used.

\section{Table 3: PAMS evaluation software}

\section{$\mathbf{1}^{\text {st }}$ Level Groundprocessingsoftware-Part of PAMS}

\begin{tabular}{l|l}
\hline QuickMosaic - On-site & Quality control on site possible
\end{tabular} right after flight

AirMosaic - Basic user A so called „dirty mosaic“ allows software after the flight

a geo-referencing of the images flown.

$2^{\text {nd }}$ level Groundprocessingsoftware - Special software not part of PAMS for orthophoto generation via server.

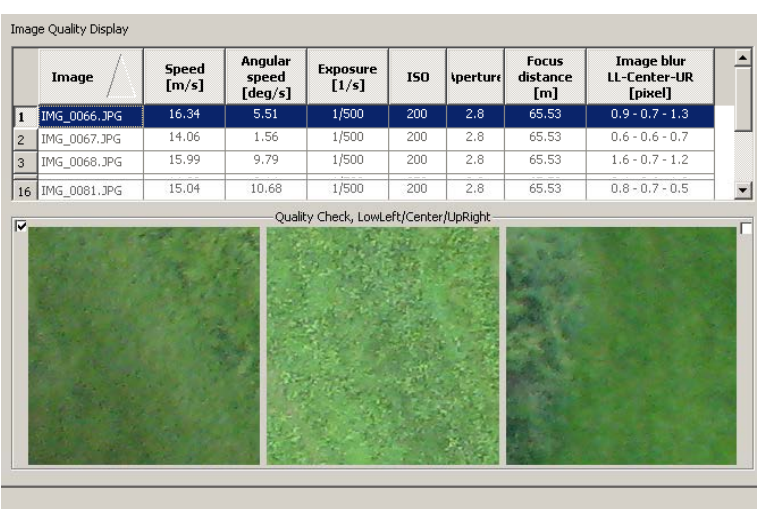

Figure 2: Image quality check in AirMosaic software. The table lists different parameters and the estimated image blur (LL-Center-UR) for each image. The visual inspection of image blur is supported by the 3 image patches used.

\subsection{Test areas}

For a first evaluation of various items, the derived data sets of the three flights given in Table 4 were used.

Table 4: Overview on test data sets.

\begin{tabular}{|c|c|c|c|}
\hline & BezF1 & BezF6 & BezF7 \\
\hline Day & March 23, 2010 & \multicolumn{2}{|c|}{ June 8, 2010} \\
\hline Strips & 14 & 8 & 8 \\
\hline Images/strip & 10 & 13 & 10 \\
\hline $\begin{array}{l}\text { Images } \\
\text { Available }\end{array}$ & 140 & 88 & 68 \\
\hline Direction & E-W & $\mathrm{N}-\mathrm{S}$ & $\mathrm{N}-\mathrm{S}$ \\
\hline Height & $200 \mathrm{~m}$ & $150 \mathrm{~m}$ & $150 \mathrm{~m}$ \\
\hline Area (plan) & $219 \times 343 \mathrm{~m}^{2}$ & $119 \times 250 \mathrm{~m}^{2}$ & $119 \times 250 \mathrm{~m}^{2}$ \\
\hline End-lap & \multirow{2}{*}{\multicolumn{3}{|c|}{$\begin{array}{l}80 \% \text { (planned) } \\
70 \% \text { (planned) }\end{array}$}} \\
\hline Side-lap & & & \\
\hline Time & $11 \mathrm{~min} 57 \mathrm{sec}$ & $5 \mathrm{~min} 39 \mathrm{sec}$ & $5 \mathrm{~min} 21 \mathrm{sec}$ \\
\hline
\end{tabular}




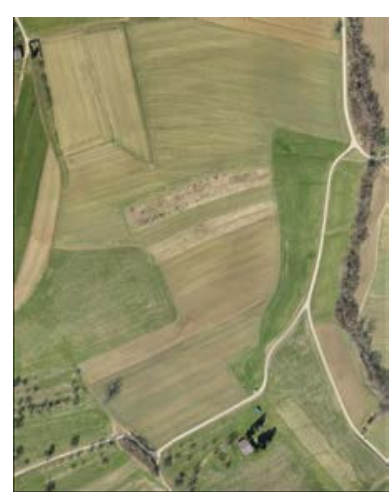

Figure 3: Flight BezF1 area.
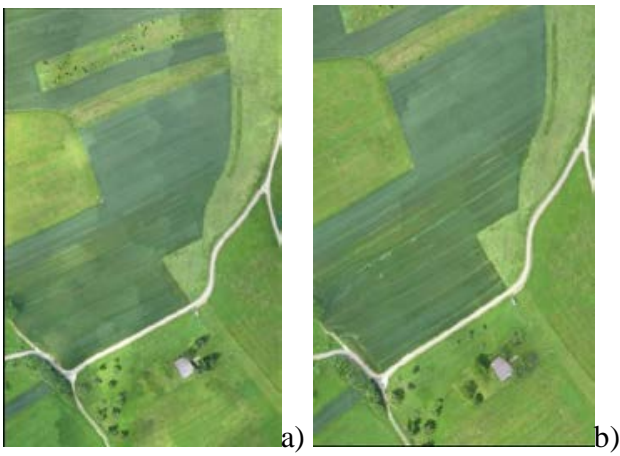

Figure 4: a) Flight BezF6 area, b) Flight BezF7 area.

The first flight (BezF1, cf. Figure 3) was performed in late March with low vegetation cover at an altitude of $200 \mathrm{~m}$ above ground and a rather big area. BezF6 covers a smaller area than flight BezF1 and was flown on June 8th 2010. The block consists of 8 strips in N-S- direction with 13 images each. The flight height was $150 \mathrm{~m}$ above ground. The flight BezF7 was performed at the same day, right after Flight BezF6. This flight was hampered by less favorable wind conditions and turbulences. For BezF6 and BezF7 the illumination conditions were very much changing due to quick moving clouds. Not all planned images could be used later on. In Figure 4 the areas of BezF6 and BezgF7 are shown, with leafs on and vegetation of various height in fields and grassland.

\subsection{Link to Trimble INPHO Software}

The HFT Stuttgart system has a PAMS Software version available, that allows the export of a text file of the output of the AirMosaic software which allows the establishment of a link to a standard photogrammetric processing chain built on Trimble INPHO software. Several obstacles had to be solved, however. The image file names on the SD card and the image Ids in the output of the AirMosaic software were not using the same naming convention. In addition the following cases were encountered on all examined test flights:

- Case 0 (normal): \# of image files per strip is the same for each strip and the total \# of image files corresponds to the total \# of image Ids from the AT in the AirMosaic SW. The linking is straightforward.

- Case 1: The number of image Ids in the AirMosaic result file is less than the number of images on the SD card, i.e. the orientation parameters of the missing images could not be derived. This image is not treated further on.

- Case 2: From the planned project orientation parameters and corresponding image file are missing.

- Case 3: A combination of Case 1 and Case 2 occurred.
The linking of the image files and image IDs and the handling the different cases (a statistics is shown in Table 5) were solved and tested on all test data sets. With the final transfer of the camera calibration file given by PAMS to the Trimble INPHO software format a standard INPHO project file can be generated, which is the basis for the further processing steps: digital aerial triangulation, DTM/DSM generation and finally digital orthomosaik generation.

Table 5: Overview on problem cases. Case 1: Missing orientation parameters but existing image file. Case 2: Missing orientation parameters AND missing image files.

\begin{tabular}{|l|c|c|c|}
\hline & BezF1 & BezF6 & BezF7 \\
\hline & March 23, 2010 & \multicolumn{2}{|c|}{ June 8, 2010 } \\
\hline Case 1 & 10 & 4 & 0 \\
\hline Case 2 & 0 & 9 & 13 \\
\hline
\end{tabular}

\subsection{Estimating image blur (BlurMetric)}

In Ziegler, 2011) the integration of the Image BlurMetric Matlab software (Bao, 2009) in a quality check process (BlurMeasurement) is described. The whole content of an image is examined and a measure between 0 and 1 for the amount of image blur is derived. For each image, according to user defined criteria, a traffic light output guides the user to the critical cases. The experiments have shown, that a range of $0-0.2$ is judged as sharp, a range of 0.2-0.37 can be judged as moderate sharp (yellow) and 0.37-1 as unsharp.

This approach will be compared in section 4.1 with the standard PAMS quality checking possibilities in the AirMosaic software.

\section{EVALUATIONS}

Having linked the PAMS data to the Trimble INPHO software the first investigations were focusing on suitable parameter settings for aerial triangulation, DTM/DSM generation as well as orthomosaik generation and a comparison of different processing results among the different flights. For this first stage no ground truth data (GCPs) were available to simulate lowaccuracy application scenarios (Ziegler, 2011).

The second part of the evaluation includes ground truth information in the processing and for the comparisons.

\subsection{Flight lines and image quality}

The module "AirMosaic" allows a quality check, which offers detailed information to the flight and to each aerial image. Especially the part on image blur is valuable information for the user to manually decide if an image is to be excluded from further processing due to image blur. The software focuses on three selected parts of the image. The effect of strong angular movements in kappa is mostly visible at the corner patches. however, those areas could just include low quality image textures in an otherwise very good image. The "blur" values should be as low as possible. The test data images had a blur range from 0.1 pel (sharp) to 7.2 pel (unsharp). No value was found to be suitable for all data sets and the software suggested too many images to be deleted, with the risk of producing gaps in the coverage. With the developed BlurMeasurment process better results could be reached. Figure 5 shows three images patches judged green, yellow and red by the BlurMetric approach. The user will have to basically look only on images judged yellow, if they shall be used for further processing or not. In four test flights it has been found, that only 4 images in average were judged RED, about 33\% of the remaining ones judged YELLOW and about $67 \%$ as GREEN. 


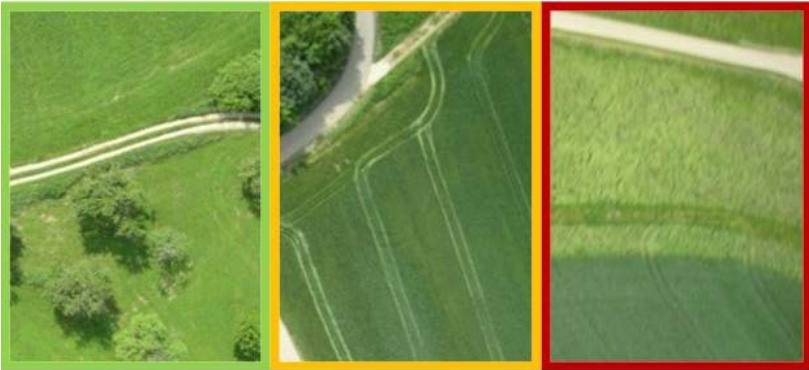

Figure 5: Three images patches with sharpness values. Left: low image blur (0.18, green), middle: moderate image blur (0.36 yellow) and high image blur $(0.38$, red $)$.

This means only about $1 / 3$ of the images marked had to be checked. Visual control and comparison to the PAMS SW on all test flights showed a more reliable behavior of the BlurMetric approach. It was found useful not to lose too many images right from the beginning, which increases the risk for gaps in the data.

\subsection{Direct geo-referencing, without ground control}

For the photogrammetric processing steps the default parameter settings for aerial imagery had to be adapted partly to the UAV flight conditions. At the beginning of the evaluations (Ziegler, 2011) for none of the three test areas GCP ground data was available. The results are purely based on the GPS information from the plane and the aerial triangulation. No manual tie point measurements or editing has been performed on the results in sections 4.2 and 4.3 .

\subsubsection{Aerial triangulation}

The usual parameters for classical digital aerial triangulation had to be adapted first. There is a problem with strong kappa angle variations from image to image and also phi and omega are partly far off from a 0 degree angle. In addition there is the problem of image noise and blur and rather big height differences from one exposure to the next one.

Table 6: MATCH-AT - Strategy and matching parameters

\begin{tabular}{|l|l|}
\hline Point density & dense \\
\hline TPC pattern: & $3 \times 3$ or $4 \times 4$ \\
\hline Start at overview level: & 6 \\
\hline Stop at overview level: & 1 or 0 \\
\hline Size of tie-point area: & 45 Pixel \\
\hline Parallax bound: & 15 Pixel \\
\hline LSM/FBM correlation coeff. & $90 \%-93 \%$ \\
\hline
\end{tabular}

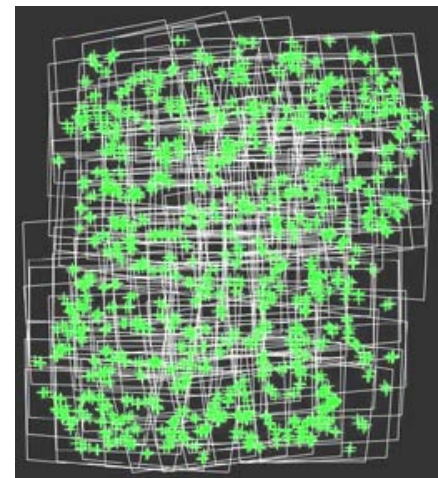

a) BezF1

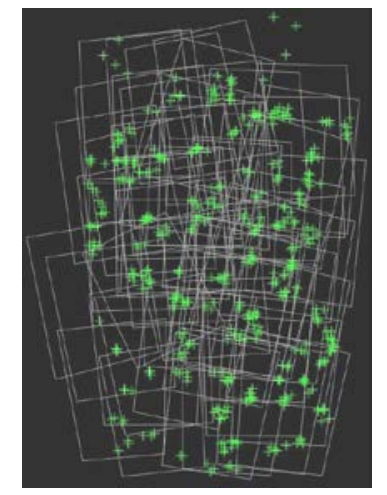

b) BezF7
Figure 6: AT tie point distribution and image footprints.
The unusual flight pattern following the mode of an ice hall sweeper with long time differences between neighbouring strips eventually causes strong illumination differences. The images are rather small and the imported attitude data is of quite low quality, as no IMU is involved.

The parameter sets in Table 6 showed quite suitable for all test data sets. A final run at level 0 (full resolution) did not bring a real improvement. The flight lines and the tie point distribution after the aerial triangulation show high regularity in case of BezF1, but weak behaviour for BezF7 due to difficult illumination and wind conditions (Figure 6).

\subsubsection{DTM/DSM Generation}

Despite the, on first sight, promising results of the aerial triangulations all test data sets showed an erroneous "Dome" in the Digital Surface Model generation in the centre of the block. The effect counted up to 10 meters in height as shown for BezF1 and BezF6 (Figure 7). Even with the introduction of the GPS flight recordings into the AT the lack of GCPs in the block could not be compensated. It is more than obvious, that this effect cannot be neglected for almost all possible applications.
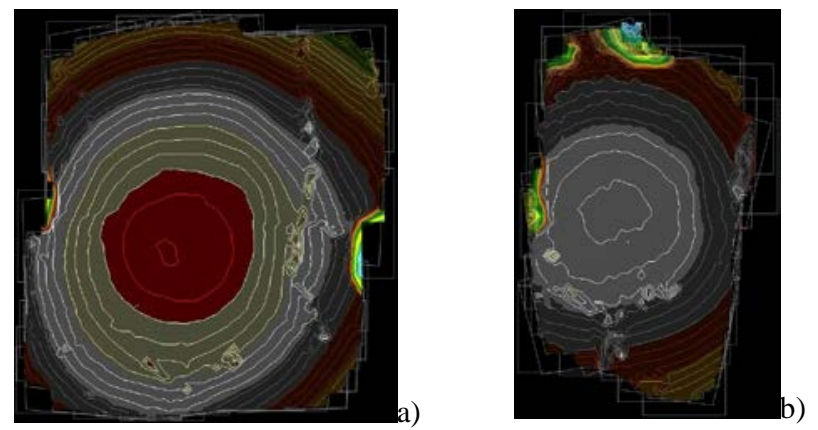

Figure 7: a) DSM of BezF1- b) DSM of BezF6. The “dome” in the center is erroneous. Contour interval $2.5 \mathrm{~m}$.

\subsubsection{Orthophoto and orthomosaik}

An orthoimage generation combined with mosaiking produced internally still consistent results. Despite the partly wrong DSM in the center of the block, the overlapping orthophotos showed only marginal deviation along road boundaries of some few $\mathrm{cm}$. Which means: for very low accuracy requirements and just visualization applications limited to a local area these results might be still usable, but not at all for real photogrammetric measuring tasks or incorporation of results in world systems.

\subsection{Ground control available}

All together 20 GCPs where measured with a LEICA GPS System 1200 ASCOS in static mode after the flight using natural points identifiable in the images. The measurement accuracy was about $5 \mathrm{~cm}$ for 19 of the measured GCPs and $50 \mathrm{~cm}$ for a height control point. The definition accuracy, however, was only about $20-30 \mathrm{~cm}$ due to the usage of nonsignalized points. In a minimum configuration only 4 GCPs were introduced into the AT, leaving 16 check points. A moderate configuration used 10 GCPs and 10 check points. The results are shown in Table 7 and Table 8 . The overall results indicate that an internal accuracy of some $10 \mathrm{~cm}$ (corresponding to about $1.5-2$ pixels) can be reached in planimetry using a moderate number of GCPs in the aerial triangulation. The $\sigma_{0}$ value is about 2 pixels only, but with $4 \mu \mathrm{m}$ still rather good. The RMS values at check points are, however, at an unacceptable range of 1 to $3 \mathrm{~m}$ due to the usage of natural and not signalized points. This does require more investigations. 
Table 7: Sigma naught and SDEV of terrain points

\begin{tabular}{|l|c|c|c|}
\hline & $\mathrm{E}[\mathrm{m}]$ & $\mathrm{N}[\mathrm{m}]$ & $\mathrm{H}[\mathrm{m}]$ \\
\hline GCP $\min$ & 0.12 & 0.11 & 0.41 \\
$\sigma_{0}=4.0 \mu \mathrm{m}$ & & & \\
\hline $\begin{array}{l}\text { GCP moderate } \\
\sigma_{0}=4.1 \mu \mathrm{m}\end{array}$ & 0.11 & 0.10 & 0.38 \\
\hline
\end{tabular}

Table 8: RMS at check points

\begin{tabular}{|l|c|c|c|}
\hline & RMS E [m] & RMS N [m] & RMS H [m] \\
\hline GCP min & 0.86 & 1.07 & 2.90 \\
\hline GCP moderate & 0.79 & 1.24 & 1.99 \\
\hline
\end{tabular}

\subsection{Comparison}

A first comparison was made between the 3 data sets without ground control. At 7 distributed points visible in all 3 data sets the deviations among the test data sets were checked. Two of them are shown in Figure 8. One can observe differences of up to 8 meters amongst the 3 flights. The results of the two flights at the same day (BezF6 and BezF7) do not really coincide. BezF1 and BezF6 show a translation (mainly $\mathrm{N}$ direction), whereas BezF7 does also show scale differences, which indicate the impact of unfavorable flight conditions.
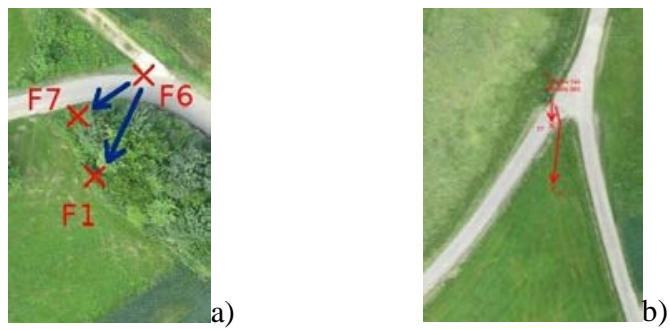

Figure 8: Results of BezF1, BezF6 and BezF7 with direct geo-referencing at two points.

Table 9: Comparison of point 9011 coordinates.

\begin{tabular}{|c|c|r|c|c|}
\hline $\begin{array}{c}\text { Point 9011 } \\
\text { (UTM32N) }\end{array}$ & $\begin{array}{c}\mathrm{E} \\
{[\mathrm{m}]}\end{array}$ & $\begin{array}{c}\text { Diff. to } \\
\text { GPS[m] }\end{array}$ & $\begin{array}{c}\mathrm{N} \\
{[\mathrm{m}]}\end{array}$ & $\begin{array}{c}\text { Diff. to } \\
\text { GPS[m } \\
]\end{array}$ \\
\hline $\begin{array}{c}\text { BezF1-no } \\
\text { GCP }\end{array}$ & 545727.06 & 14.72 & 5389787.88 & 15.41 \\
\hline $\begin{array}{c}\text { OpenStreet } \\
\text { Map }\end{array}$ & 545709.97 & -2.37 & 5389771.59 & -0.88 \\
\hline $\begin{array}{c}\text { Google } \\
\text { Maps }\end{array}$ & 545709.99 & -2.35 & 5389771.52 & -0.95 \\
\hline GPS & 545712.34 & - & 5389772.47 & - \\
\hline $\begin{array}{c}\text { BezF1 } \\
\text { moderate }\end{array}$ & 545712.25 & -0.09 & 5389772.57 & 0.10 \\
\hline
\end{tabular}
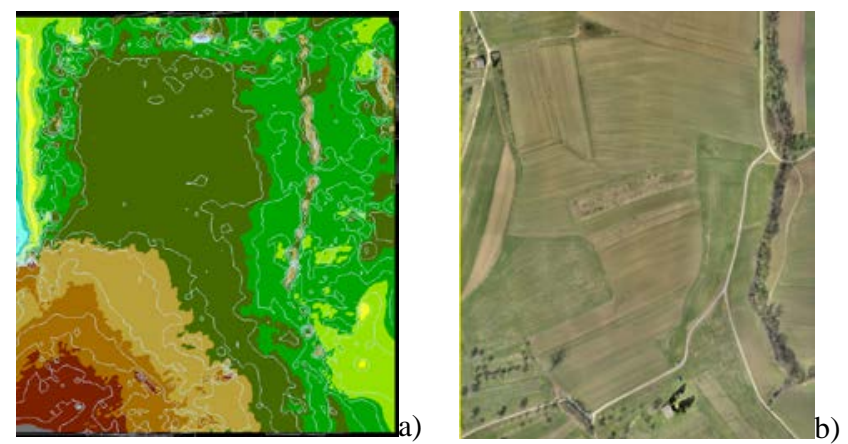

Figure 9: BezF1 with moderate \# of GCPs. a) DSM BezF1, b) Orthomosaik BezF1.
For several points a final comparison to ground truth GPS measurements was performed as well as to freely available data as OpenStreetMap and Google Maps. One example is given in Table 9. The flight with direct geo-referencing showed very large deviation from the ground truth GPS, whereas the AT with moderate number of GCPs showed a very good result. The results of the final run with the moderate number of GCPs produced a good result for the DSM and the orthomosaik as shown in Figure 9 for BezF1.

\section{CONCLUSIONS}

Even without editing of the aerial triangulation the results are feasible due to the selection of suitable parameter sets for this kind of aerial imagery. It is clearly proven, that no project should be flown without proper GCPs introduced into the aerial triangulation. Results indicate that an internal quality of some $\mathrm{dm}$ can be reached with a moderate number of GCPs well distributed. This still needs further investigation. The external flight conditions do influence the aerial triangulation, which might have to be supported by manual tie point measurements. The developed blur measurement process assists the user in selecting the most suitable images for the photogrammetric processing chain. Further investigations will incorporate selfcalibration in a hilly test site to check for the camera calibration parameters in flight and focus on a preliminary matching of neighbouring images to better predict the kappa rotation as input to the aerial triangulation.

\section{REFERENCES}

Bao, D., 2009. Image Blur Metric, http://www.mathworks.com/matlabcentral/fileexchange/24676image-blur-metric. TheMathWorks, Inc. (accessed November 24, 2010).

Brinker, S., 2008. Prozessentwicklung und Evaluierung für photogrammetrische Aufnahmen mit einem UAV: Diplomarbeit HFT Stuttgart.

Everaerts, J., 2006. New Platforms - Unconventional Platforms for Remote Sensing. EuroSDR Project Presentations.

Gatewing, 2011. www.gatewing.com (accessed July 24th 2011).

Germatics, 2009. www.germatics.com (accessed March 2009).

Germatics, 2011. www.germatics.com (accessed July 24th 2011).

ISPRS, 2004. Inter-Commission WG I/V - "Autonomous Vehicle Navigation 2004-2008. www.isprs.org.

ISPRS, 2011. Inter-Commission Working Group I/V - "UVS for mapping and monitoring applications" 2008-2012. www.commission1.isprs.org/icwg1_5.

Mavinci, 2011. /www.mavinci.de (accessed July 24th 2011).

Trimble, 2011. Trimble Inpho Software. www.inpho.de (accessed July 24th 2011).

Ziegler, M., 2011. Photogrammetrische Evaluation multitemporaler UAV-Aufnahmen mit Teilautomatisierung des Auswertungsprozesses, Bachelorarbeit HFT Stuttgart.

\section{ACKNOWLEDGEMENTS}

The thesis works by Dipl-Ing. (FH) Sören Brinker and BSc. Marius Ziegler have been the basis for this paper and their contribution is gratefully acknowledged. 